\title{
Estrogen-related receptor $\gamma$ promotes the migration and metastasis of endometrial cancer cells by targeting S100A4
}

\author{
TENG HUA $^{1 *}$, XIAOXIAO WANG ${ }^{1 *}$, SHUQI CHI ${ }^{1}$, YAN LIU ${ }^{1}$, DILU FENG ${ }^{1}$, \\ YINGCHAO ZHAO $^{2}$ and HONGBO WANG ${ }^{1}$ \\ ${ }^{1}$ Department of Obstetrics and Gynecology, and ${ }^{2}$ Cancer Center, Union Hospital, Tongji Medical College, \\ Huazhong University of Science and Technology, Wuhan, Hubei 430023, P.R. China
}

Received November 28, 2017; Accepted May 15, 2018

DOI: 10.3892/or.2018.6471

\begin{abstract}
S100 calcium binding protein A4 (S100A4) is a well-established tumor metastasis mediator in various malignancies, including endometrial cancer (EC). However, the regulatory mechanism underlying S100A4 expression remains elusive. In the present study, by analyzing public datasets and clinical samples, we found that estrogen-related receptor $\gamma(\mathrm{ERR} \gamma)$ was upregulated and positively correlated with S100A4 transcription in EC. ERR $\gamma$ knockdown inhibited S100A4 expression and promoted the expression of its downstream target E-cadherin, and vice versa. Mechanistic studies indicated that ERR $\gamma$ enhanced the promoter activity of S100A4 to facilitate its transcription. In addition, knockdown of ERR $\gamma$ suppressed migration and invasion of EC cells in vitro, while ectopic ERR $\gamma$ expression promoted migration and invasion of EC cells in vitro and tumor growth in vivo. Importantly, restoration of S100A4 expression prevented EC cells from undergoing ERR $\gamma$-mediated changes in these biological features. In addition, synchronous changes in S100A4 and ERR $\gamma$ expression were observed after incubation with estrogen. Overall, ERR $\gamma$ may exert oncogenic activity mainly associated with aggressiveness of EC by activating S100A4 transcription and thus may be a novel therapeutic target in EC.
\end{abstract}

Correspondence to: Professor Hongbo Wang, Department of Obstetrics and Gynecology, Union Hospital, Tongji Medical College, Huazhong University of Science and Technology, 1277 Jiefang Avenue, Wuhan, Hubei 430022, P.R. China

E-mail: hb_wang1969@sina.com

Professor Yingchao Zhao, Cancer Center, Union Hospital, Tongji Medical College, Huazhong University of Science and Technology, 156 Wujiadun Road, Hankou, Wuhan, Hubei 430023, P.R. China

E-mail: 2006xh0836@hust.edu.cn

*Contributed equally

Key words: endometrial cancer, ERR $\gamma$, invasion, metastasis, S100A4

\section{Introduction}

Endometrial cancer (EC) is the most common gynecologic tumor in developed countries, with an increasing prevalence (1). It is estimated that 61,380 new cases and 10,920 deaths resulting from EC occurred in 2017 (2). Adenocarcinoma of the endometrium accounts for over $70 \%$ of all uterine cancers. Most patients $(80 \%)$ are diagnosed with early disease and can be surgically cured. However, the outcome of advanced or recurrent cases remains far worse, and the adjuvant treatment options are quite limited (1). Discovery of novel targets is warranted to better understand the EC pathogenesis and to develop new therapeutic approaches for this disease. It is well known that tumor progression and metastasis are often linked to epithelial-mesenchymal transition (EMT). During this process, a more invasive cell phenotype is established, accompanied by alterations in the expression of many core molecules, particularly E-cadherin (3). Thus, it is worthwhile to explore the regulatory mechanism of EMT in the tumor biology of EC.

S100 calcium binding protein A4 (S100A4) has been shown to be involved in biological functions that contribute to malignant tumors, such as proliferation, apoptosis, metastasis, angiogenesis and immune evasion (4). More importantly, S100A4 plays pivotal roles in tumor invasion by triggering EMT, mechanically serves as a downstream target gene of the wnt $/ \beta$-catenin pathway, modulates membrane integrin signaling, and directly promotes cell motility through interaction with cytoskeletal proteins such as myosin, actin and tropomyosin (5-7). Elevated S100A4 expression has been found in many types of tumors and is closely related to poor outcome in tumor patients (8). Our previous research revealed that S100A4 is highly expressed in EC cells, and knockdown of S100A4 expression resulted in suppression of the migration and invasion capability of EC cells, which may partially occur via EMT-related modifications (9). However, the regulatory mechanisms of $\mathrm{S} 100 \mathrm{~A} 4$ expression in $\mathrm{EC}$ remain to be elucidated.

Estrogen-related receptors (ERRs; ERR $\alpha, E R R \beta$ and ERR $\gamma$ ) comprise a subgroup of orphan nuclear receptors that share highly homologous DNA-binding domains with 
estrogen receptors. However, ERRs do not bind to endogenous estrogens or their derivatives, and thus are designated orphan receptors (10). High ERR $\gamma$ expression levels are often associated with high metabolic demand in human tissues, such as skeletal muscle, heart and brown adipose tissue. Accumulating evidence indicates a central role of ERR $\gamma$ in metabolic genes and cellular energy metabolism regulation (11). Apart from metabolic disease, recent studies have revealed the clinical significance of ERR $\gamma$ in several cancer types, including EC. In breast cancer, ERR $\gamma$ is generally overexpressed and related to lymph node status and upregulated during tamoxifen resistance acquisition, indicating a cancer promoting role of ERR $\gamma(12,13)$. The role of ERR $\gamma$ in EC remains unclear. Overexpression of ERR $\gamma$ is correlated with increased clinical stage, deeper myometrium invasion and positive lymph node status $(14,15)$. In addition, ERR $\gamma$ mediates estrogen-induced proliferation of EC cells (16). However, the effect of ERR $\gamma$ on EC cell migration and metastasis has never been explored.

In the present study, augmented expression of ERR $\gamma$ was found, and for the first time, a correlation between ERR $\gamma$ and S100A4 expression was identified in clinical EC tissues via experimental techniques and public database mining. Furthermore, ERR $\gamma$ directly facilitates S100A4 transcription through promoter activation, thus promoting migration and invasion of EC cells both in vitro and in vivo, demonstrating the emerging roles of ERR $\gamma$ in EC progression through transcriptional regulation of S100A4.

\section{Materials and methods}

Patients and specimens. The present study was performed in accordance with the Declaration of Helsinki, and approval to conduct the present study was obtained from the Ethics Committee of Tongji Medical College, Huazhong University of Science and Technology (IORG no. IORG0003571). Tissues were collected after receiving informed consent from the patients. Formalin-fixed paraffin-embedded specimens were obtained from 20 primary EC patients [age (mean $\pm \mathrm{SD}$ ), $51.5 \pm 12.1$ years] who had undergone surgical resection at WuHan Union Hospital between September 2015 and December 2016. Those that had already received adjuvant therapy, such as chemotherapy, hormone therapy or radiotherapy, were excluded. Fresh specimens from the above-mentioned EC patients and another 20 normal endometrium cases (age, $49.6 \pm 9.4$ years) were collected for protein and total RNA extraction and stored in liquid nitrogen until use.

Immunohistochemical staining. Fresh specimens were fixed in $10 \%$ formaldehyde for at least $24 \mathrm{~h}$ and embedded in paraffin. Tissue slides with $4-\mu \mathrm{m}$-thick sections were constructed and dewaxed in xylene and rehydrated in a graded alcohol series. Antigen retrieval was conducted by heating slides in $0.01 \mathrm{M}$ of sodium citrate buffer for $20 \mathrm{~min}$. Endogenous non-specific peroxidase activity was blocked with $3 \% \mathrm{H}_{2} \mathrm{O}_{2}$ for $15 \mathrm{~min}$, and non-specific staining was blocked by incubation with $10 \%$ normal goat serum for $30 \mathrm{~min}$. Then, the samples were incubated with $200 \mu$ l of primary antibodies against ERR $\gamma(1: 400$; cat. no. ab49129; Abcam, Cambridge, MA, USA), S100A4 (1:400; cat.no. 13018S; Cell Signaling Technology, Inc., Beverly,
MA, USA) and Ki-67 (1:200; cat. no. sc-23900; Santa Cruz Biotechnology, Inc., Santa Cruz, CA, USA) at $4^{\circ} \mathrm{C}$ overnight. After being washed with phosphate-buffered saline (PBS), the slides were incubated with EnVision/HRP, rabbit secondary antibody (1:1,000; cat. no. GB23303; Servicebio Technology, Co., Ltd., Wuhan, China) for $30 \mathrm{~min}$. Diaminobenzidine substrate was used for visualization, followed by counterstaining with hematoxylin. Finally, the slides were dehydrated and mounted. The immunohistochemistry scoring strategy was performed as previously described (9).

Western blot analysis. Collected fresh EC tissues or cultured EC cells were washed with ice-cold PBS 3 times and lysed with radio-immunoprecipitation assay buffer containing protease inhibitors. The protein concentration was determined using a bicinchoninic acid (BCA) protein assay kit. Equal amounts of proteins $(30 \mu \mathrm{g})$ were added to $12 \%$ SDS-PAGE gels and transferred onto nitrocellulose membranes. After being blocked with $5 \%$ skim milk in $1 \mathrm{X}$ TBS buffer containing $0.1 \%$ Tween-20 at room temperature for $1 \mathrm{~h}$, the membranes were incubated with primary antibodies against ERR $\gamma$ (1:800; cat. no. ab49129; Abcam), S100A4 (1:800; cat. no. 13018S; Cell Signaling Technology), GAPDH (1:1,000; cat. no. sc-66163; Santa Cruz Biotechnology) and E-cadherin (1:200; cat. no. sc-52327; Santa Cruz Biotechnology) at $4^{\circ} \mathrm{C}$ overnight. The target proteins were visualized using enhanced chemiluminescence reagents (Thermo Fisher Scientific, Inc., Waltham, MA, USA) after incubation with goat anti-rabbit secondary antibody (1:5,000; cat. no. sc-2004; Santa Cruz Biotechnology). Enhanced chemiluminescence reagents (Thermo Fisher Scientific) were used for bands detection. The optical density was quantified using Bio-Rad Image $\mathrm{Lab}^{\mathrm{TM}}$ v4.1 software.

Real-time quantitative RT-PCR. Total RNA from EC tissues and cultured EC cells was isolated with RNAiso Plus (Takara Bio, Co., Ltd., Otsu, Japan) following the manufacturer's protocol. The reverse transcription reactions were carried out using a PrimeScript RT reagent kit (Takara Bio). The primers involved were as follows: ERR $\gamma$ (102 bp), 5'-CCCGACAGT GACATCAAAGCC-3' (sense) and 5'-CGTGGAGAAGCC TGGAATATGC-3' (antisense); S100A4 (251 bp), 5'-TACTCG GGCAAAGAGGGTGA-3' (sense) and 5'-CATTTCTTCCTG GGCTGCTTA-3' (antisense); E-cadherin (162 bp), 5'-GAG AACGCATTGCCACATACAC-3' (sense) and 5'-GAGCAC CTTCCATGACAGACCC-3' (antisense); GAPDH (255 bp), 5'-ACTTTGGTATCGTGGAAGGACTAT-3' (sense) and 5'-GTTTTTCTAGACGGCAGGTCAGG-3' (antisense). Real-time PCR was conducted with Premix Ex Taq (Takara Bio), as previously described (9). The $2^{-\Delta \Delta \mathrm{Ct}}$ method was employed for relative transcript abundance determination. Each experiment was performed in triplicate at least 3 times.

Cell culture and transfection. The human EC cell lines Ishikawa, AN3CA, HEC-1A and HEC-1B were purchased from the American Type Culture Collection (ATCC, Manassas, VA, USA). Cells were cultured in appropriate medium supplemented with $10 \%$ fetal bovine serum (FBS), $100 \mathrm{U} / \mathrm{ml}$ of penicillin and $100 \mu \mathrm{g} / \mathrm{ml}$ of streptomycin. All the cells were incubated at $37^{\circ} \mathrm{C}$ in a humidified atmosphere containing $5 \% \mathrm{CO}_{2}$. 
Table I. Oligonucleotide sets used for constructs and short hairpin RNAs.

\begin{tabular}{ll}
\hline Oligo set & \multicolumn{1}{c}{ Sequences } \\
\hline sh-Scb & 5'-CCGGTTCTCCGAACGTGTCACGTTTCAAGAGAACGTGACACGTTCGGAGAATTTTTG-3' (sense) \\
& 5'-AATTCAAAAATTCTCCGAACGTGTCACGTTCTCTTGAAACGTGACACGTTCGGAGAA-3' (antisense) \\
sh-ERR $\gamma$-1 & 5'-CCGGCCTCACTACACTGTGTGACTTCTCGAGAAGTCACACAGTGTAGTGAGGTTTTTG-3' (sense) \\
& 5'-AATTCAAAAACCTCACTACACTGTGTGACTTCTCGAGAAGTCACACAGTGTAGTGAGG-3' (antisense) \\
sh-ERR $\gamma$-2 & 5'-CCGGCGAATGAATGTGAAATCACAACTCGAGTTGTGATTTCACATTCATTCGTTTTTG-3' (sense) \\
& 5'-AATTCAAAAACGAATGAATGTGAAATCACAACTCGAGTTGTGATTTCACATTCATTCG-3' (antisense) \\
sh-S100A44 5'-CCGGCGCCATGATGTGTAACGAATTCTCGAGAATTCGTTACACATCATGGCGTTTTTG-3' (sense) & 5'-AATTCAAAAACGCCATGATGTGTAACGAATTCTCGAGAATTCGTTACACATCATGGCG-3' (antisense)
\end{tabular}

ERR $\gamma$, estrogen-related receptor $\gamma$; S100A4, S100 calcium binding protein A4; sh-Scb, scramble short hairpin RNA.

Plasmid/construct transfection. Oligonucleotides encoding short hairpin RNAs (shRNAs) for ERR $\gamma$ (sh-ERR $\gamma$ ), S100A4 (sh-S100A4), scramble shRNA (sh-Scb), and S100A4 expression cDNA (GV230-S100A4) were purchased from Shanghai GeneChem (Shanghai, China). Invitrogen ${ }^{\mathrm{TM}}$ Lipofectamine 2000 (Thermo Fisher Scientific) was used for transfection. The target sequences for synthetic oligonucleotide primers are listed in Table I. A lentiviral vector carrying ERR $\gamma$ gene fragments was constructed to upregulate ERR $\gamma$ expression in HEC-1A and AN3CA cells. The empty vector served as the control. Stable cell lines were selected by administration of geneticin or puromycin (Sigma-Aldrich; Merck KGaA).

Luciferase reporter assay. Sequences containing different S100A4 promoter sections were cloned and sub-cloned to pcDNA3.0 basic vectors. EC cells were plated on 24-well plates overnight and co-transfected with luciferase reporter vectors and the internal control plasmid pRL-SV40 (Promega Corp., Madison, WI, USA) carrying the Renilla luciferase gene for 24 h. Firefly and Renilla luciferase activities were measured with a dual-luciferase assay system kit (Promega Corp.). Experiments performed for the analysis were repeated at least 3 times.

Transwell migration and invasion assays. Migration assays were performed using Transwell inserts with $8.0-\mu \mathrm{m}$ pore membrane filters (Corning Costar, Tewksbury, MA, USA). For invasion assays, the microfilters were precoated with $50 \mu 1$ of Matrigel matrix (BD Biosciences, Sparks, MD, USA). Then, $5 \times 10^{4}$ homogeneous single cells were plated in the upper chambers of each well with low-serum medium (1\% FBS), and a chemo-attractant (medium containing $10 \%$ FBS) was added to the lower chamber, followed by a $24-\mathrm{h}$ incubation. Afterwards, the membranes were fixed with $4 \%$ paraformaldehyde and stained with $0.5 \%$ crystal violet. The numbers of migrating and invading cells were determined in 5 random fields of each membrane using CX23 Olympus light microscopy (Olympus Optical Co., Ltd., Tokyo, Japan). Three replicates were performed for this analysis.

In vivo tumor growth assay. All animal experiments were approved by the Animal Care Committee of Tongji Medical
College. Female 4- to 6-week-old and weight $20 \mathrm{~g} \mathrm{BALB/c}$ nude mice were purchased from Vital River Laboratory Animal Technology Co., Ltd. (Beijing, China). The animals were housed at $23 \pm 1^{\circ} \mathrm{C}$ under a standard $12 \mathrm{~h} / 12 \mathrm{~h}$ light-dark cycle with free access to food and water. Mice were injected subcutaneously in the right flank with $5 \times 10^{6}$ HEC-1A cells stably transfected with ERR $\gamma$ or empty vectors as indicated ( $n=5$ per group). Tumor volume (V) was measured every 4 days and calculated via the formula: $\mathrm{V}=$ length $\mathrm{x}$ width ${ }^{2} / 2$. At 42 days after injection, the mice were sacrificed, and subcutaneous tumors were weighed and photographed using a camera. The protein levels of ERR $\gamma, \mathrm{S} 100 \mathrm{~A} 4$ and Ki-67 were analyzed in tumor tissues via immunohistochemistry.

Statistical analysis. Statistical analysis was performed using GraphPad Prism v5.0 statistical software (GraphPad Software, Inc., La Jolla, CA, USA). All results are expressed as the mean \pm standard error of the mean (SEM). Differences among groups were determined with Student's t-test or one-way analysis of variance (ANOVA). The post hoc test was performed for intergroup comparison of parametric data. Pearson's coefficient correlation was applied to investigate the association between ERR $\gamma$ and S100A4 expression levels. P-values $<0.05$ were statistically significant.

\section{Results}

ERR $\gamma$ is highly expressed and positively correlated with S100A4 expression in EC tissues. Mining the publicly available databases Gene Expression Omnibus website (GEO; http://www.ncbi.nlm.nih.gov/geo/) and R2, microarray analysis and visualization platform (http://hgserverl.amc. $\mathrm{nl} /$ cgi-bin/r2/main.cgi) revealed increased ERR $\gamma$ transcription levels in EC compared with benign endometrium (Fig. 1A), and an inverse correlation between ERR $\gamma$ and S100A4 transcription levels was observed in several types of cancers, including non-small cell lung and colon cancer, squamous cell carcinoma of the tongue, oral cavity cancer, and Wilms' tumors (data not shown). To investigate ERR $\gamma$ expression in EC, fresh tissues from 20 well established primary EC patients and 20 normal endometrium cases were collected. Real-time quantitative RT-PCR and western blotting showed higher 
A

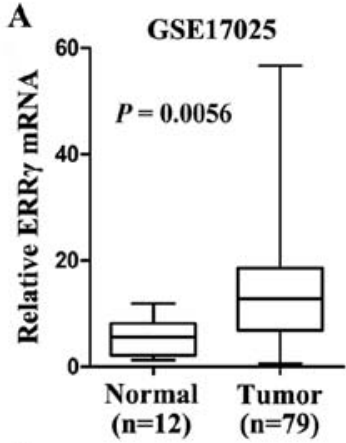

C

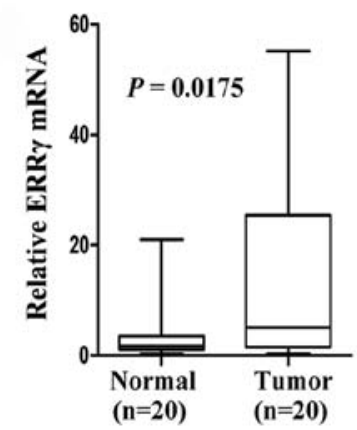

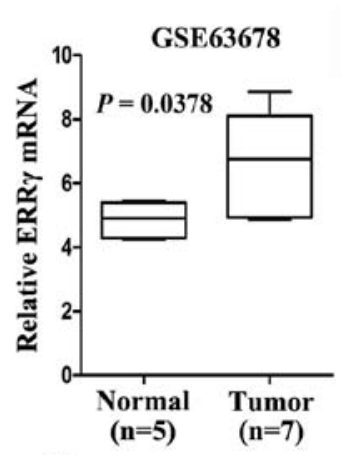

D

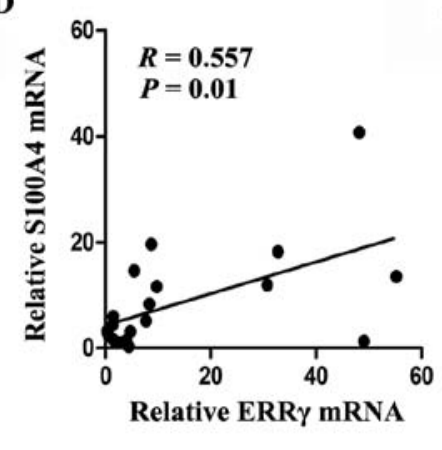

B

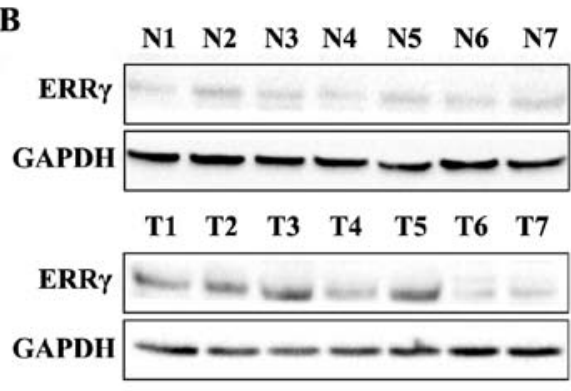

$\mathbf{E}$

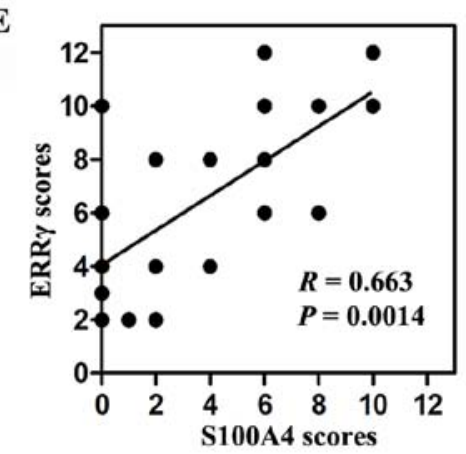

$\mathbf{F}$

Tumor 1

Tumor 2
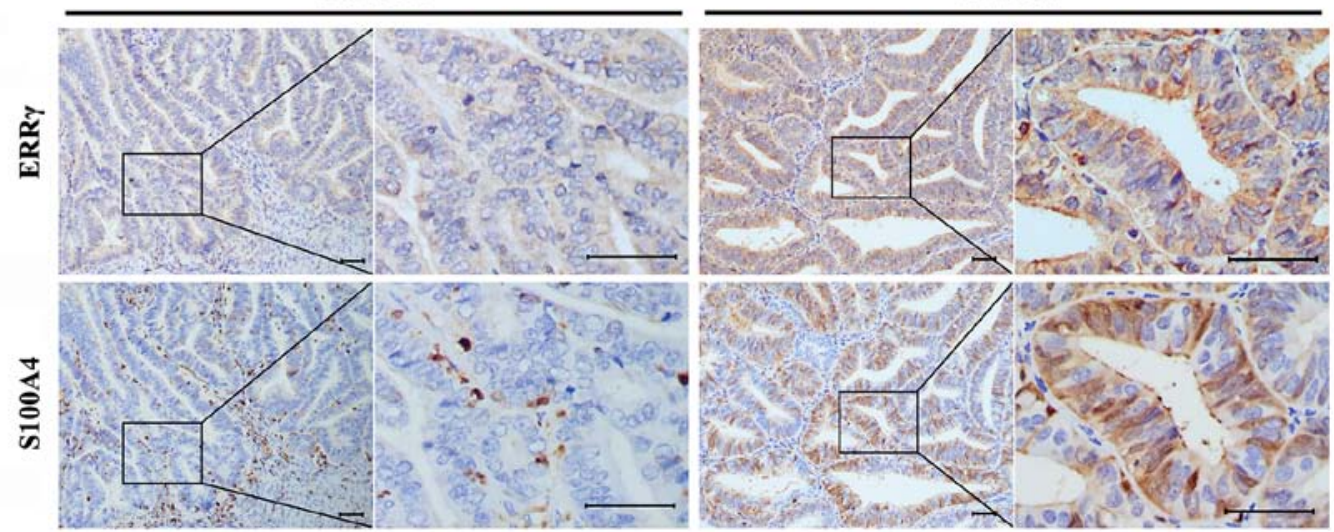

Figure 1. ERR $\gamma$ is highly expressed and positively correlated with S100A4 expression in EC tissues. (A) Transcription levels of ERR $\gamma$ in endometrial cancer (tumor) and benign endometrium (normal) tissues from GEO databases (GSE17025 and GSE63678) (38,39). (B) Representative western blots of ERR $\gamma$ protein from normal endometrium (N) and endometrial cancer (T). (C) The mRNA levels of ERR $\gamma$ in endometrial cancer (tumor) and benign endometrium (normal) tissues as indicated by RT-qPCR. (D) Correlation of ERR $\gamma$ and S100A4 transcription levels in endometrial cancer patients. (E) Positive correlation between the ERR $\gamma$ and S100A4 staining scores in EC tissues. (F) Representative immunohistochemical staining of ERR $\gamma$ and S100A4 (brown) in EC tissues. Scale bars, $50 \mu \mathrm{m}$. S100A4, S100 calcium binding protein A4; ERR $\gamma$, estrogen-related receptor $\gamma$.

expression levels of ERR $\gamma$ in EC specimens than the levels noted in the benign endometrium (Fig. $1 \mathrm{~B}$ and $\mathrm{C}$ ). In addition, a positive correlation between ERR $\gamma$ and S100A4 transcription levels in EC tissues was verified with real-time quantitative RT-PCR (correlation coefficient $\mathrm{R}=0.557, \mathrm{P}=0.01$, Fig. 1D) and immunohistochemical staining (correlation coefficient $\mathrm{R}=0.663, \mathrm{P}=0.0014$, Fig. $1 \mathrm{E}$ and $\mathrm{F}$ ). These results indicated that ERR $\gamma$ is overexpressed and positively correlated with S100A4 in EC patients.

ERR $\gamma$ transcriptionally regulates the expression of S100A4 in cultured EC cells. The transcription levels of ERR $\gamma$ are low in most EC cell lines, as indicated by the Cancer Cell Line Encyclopedia (CCLE) program (http://www.broadinstitute. org/ccle; Fig. 2A), and this finding was validated by western blotting and real-time quantitative RT-PCR in 4 representative
EC cell lines (Fig. 2B and C). Expression of ERR $\gamma$ was relatively high in $\mathrm{HEC}-1 \mathrm{~B}$ cells but almost undetectable in Ishikawa, HEC-1A and AN3CA EC cells. To explore the hypothesis that ERR $\gamma$ may modulate the expression of S100A4 in EC, HEC-1B cells were stably transfected with sh-ERR $\gamma$, leading to decreased protein and transcription levels of ERR $\gamma$ and S100A4 compared to those in cells transfected with sh-Scb (Fig. 2D and E). Additionally, expression of the S100A4 downstream gene E-cadherin was significantly upregulated in ERR $\gamma$-silenced EC cells. As restoration of S100A4 partially abolished the impact of ERR $\gamma$ on E-cadherin expression (data not shown), we believed that ERR $\gamma$ regulated E-cadherin expression through S100A4 in EC cells. Inversely, stable transfection of HEC-1A and AN3CA EC cells with ERR $\gamma$ notably upregulated and downregulated the expression of S100A4 and E-cadherin, respectively, compared with 

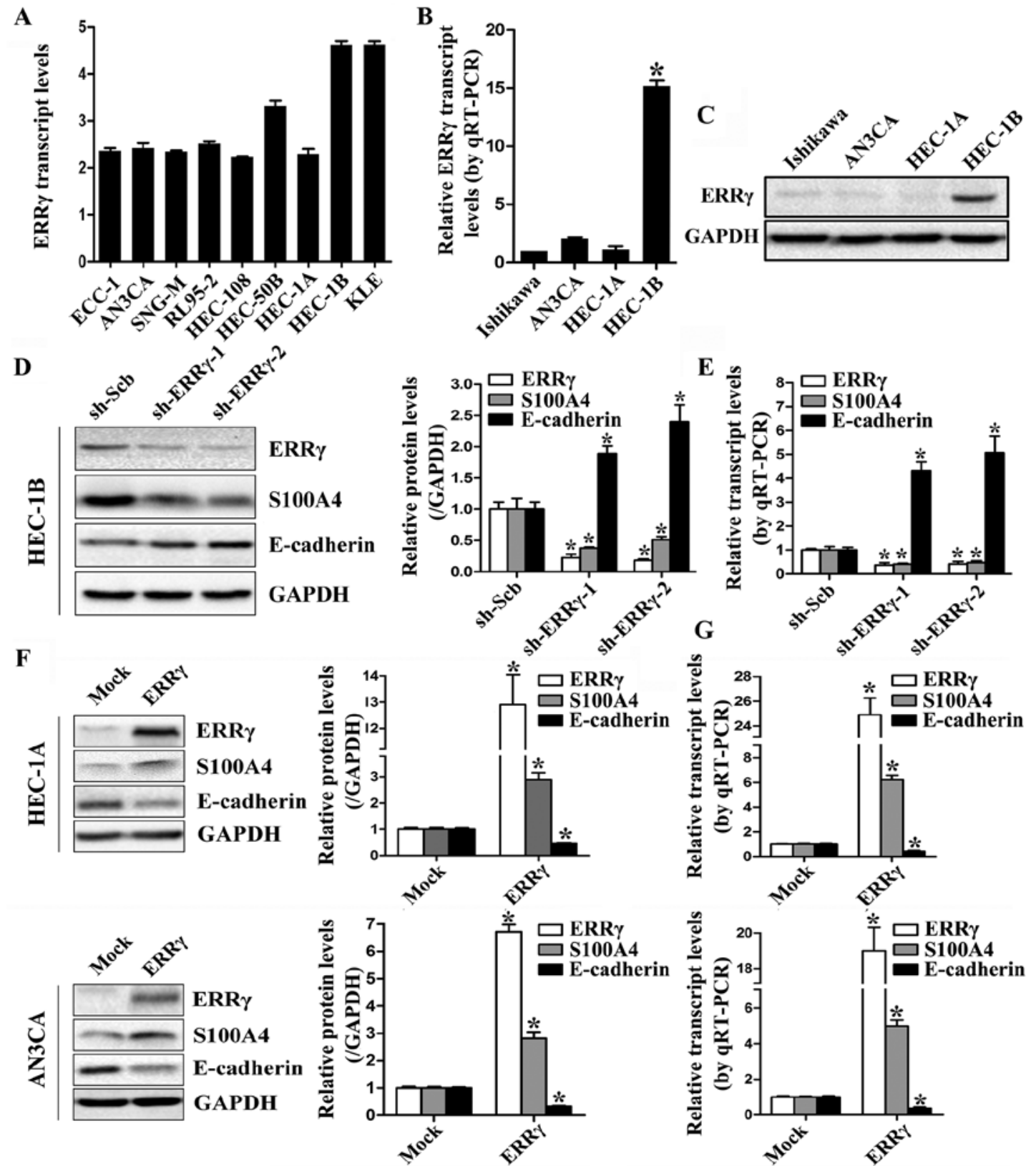

Figure 2. ERR $\gamma$ regulates the expression of S100A4 in cultured EC cell lines. (A) Transcription levels of ERR $\gamma$ in 9 EC cell lines extracted from the Cancer Cell Line Encyclopedia (CCLE) program (http://www.broadinstitute.org/ccle). (B and C) qRT-PCR and western blotting showing the mRNA and protein levels of ERR $\gamma$ in Ishikawa, AN3CA, HEC-1A and HEC-1B cells. (D and E) Western blotting and qRT-PCR results indicating the ERR $\gamma$, S100A4 and E-cadherin protein and mRNA levels in HEC-1B cells transfected with scramble short hairpin RNA (sh-Scb) or sh-ERR $\gamma$. (F and G) Western blotting and qRT-PCR results indicating the ERR $\gamma, \mathrm{S} 100 \mathrm{~A} 4$ and E-cadherin protein and mRNA levels in HEC-1A (top) and AN3CA (bottom) cells transfected with empty vector (mock) and ERR $\gamma$. ${ }^{*}<0.05$. S100A4, S100 calcium binding protein A4; ERR $\gamma$, estrogen-related receptor $\gamma$.

cells transfected with the empty vector (Fig. $2 \mathrm{~F}$ and G). These results demonstrated that ERR $\gamma$ could modulate the expression of S100A4 in EC cells.

To investigate whether ERR $\gamma$ could transcriptionally increase S100A4 expression, computational assessment from the JASPAR CORE Database (http://jaspar.genereg.net) revealed three potential binding sites of ERR $\gamma$ within the S100A4 promoter, located 368-377, 639-648 and 731-740 bp upstream of the transcription start site (TSS). An S100A4 promoter luciferase reporter and its truncation vectors were constructed and used to transfect EC cells. A dual-luciferase assay revealed that the region $-656 /-784$ bp relative to the TSS was essential for S100A4 promoter activities, and deletion of this region resulted in remarkably decreased S100A4 promoter activities in cultured HEC-1A and AN3CA cells (Fig. 3A). Moreover, knockdown of ERR $\gamma$ in cultured HEC-1B cells attenuated the promoter activities of S100A4, and ectopic expression of ERR $\gamma$ enhanced the S100A4 promoter activities in HEC-1A and AN3CA cells (Fig. 3B and C). These results indicated that ERR $\gamma$ could trigger S100A4 transcription through promoter activation.

ERR $\gamma$ modulates the migration and invasion capability of EC cells through S100A4 in vitro. We first explored the effects of 
A

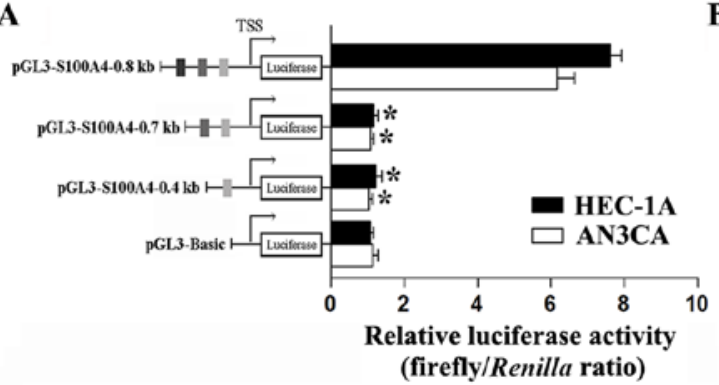

B

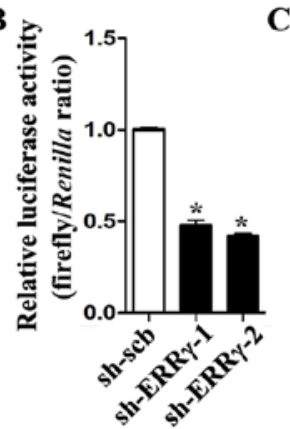

C

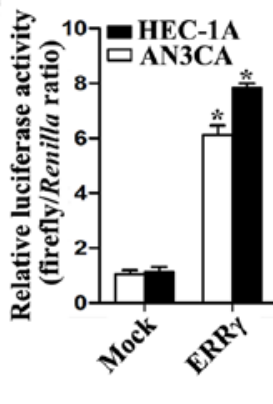

Figure 3. ERR $\gamma$ transcriptionally activates S100A4 expression in cultured EC cells. (A) Dual-luciferase assay showing the S100A4 promoter activities with different truncations in HEC-1A and AN3CA cells. (B) Dual-luciferase assay displaying the promoter S100A4 activities in HEC-1B cells transfected with sh-Scb or sh-ERR $\gamma$. (C) Dual-luciferase assay showing the S100A4 promoter activities in HEC-1A and AN3CA cells transfected with mock or ERR $\gamma$. ${ }^{*}<0.05$. S100A4, S100 calcium binding protein A4; ERR $\gamma$, estrogen-related receptor $\gamma$.

$\mathbf{A}$
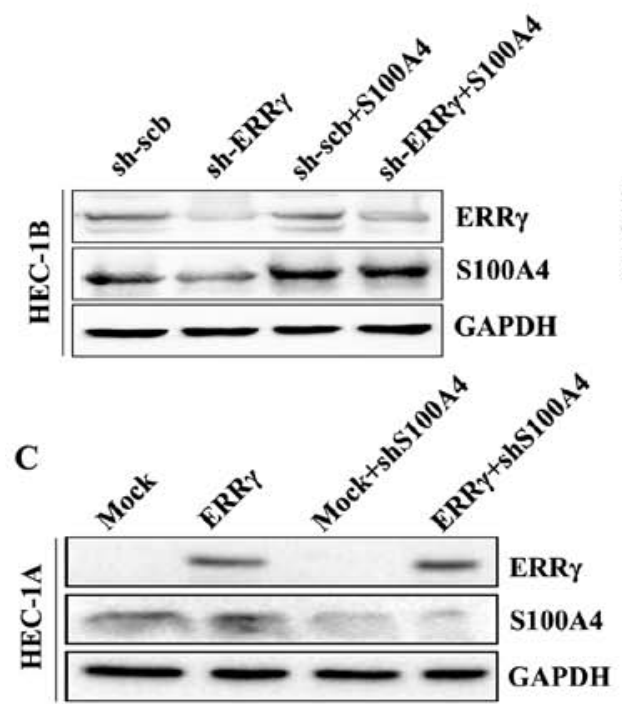

B

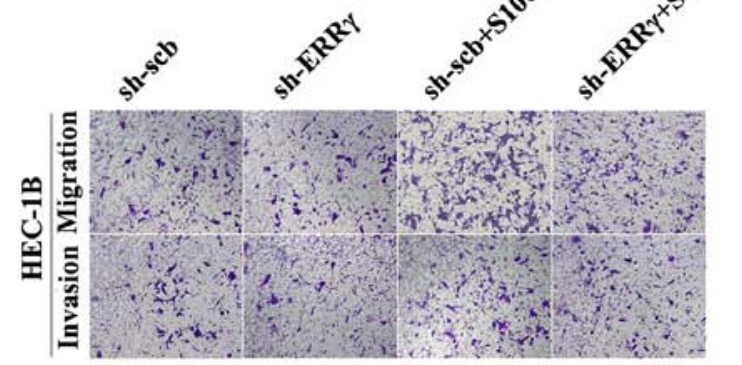

D



$\mathbf{E}$
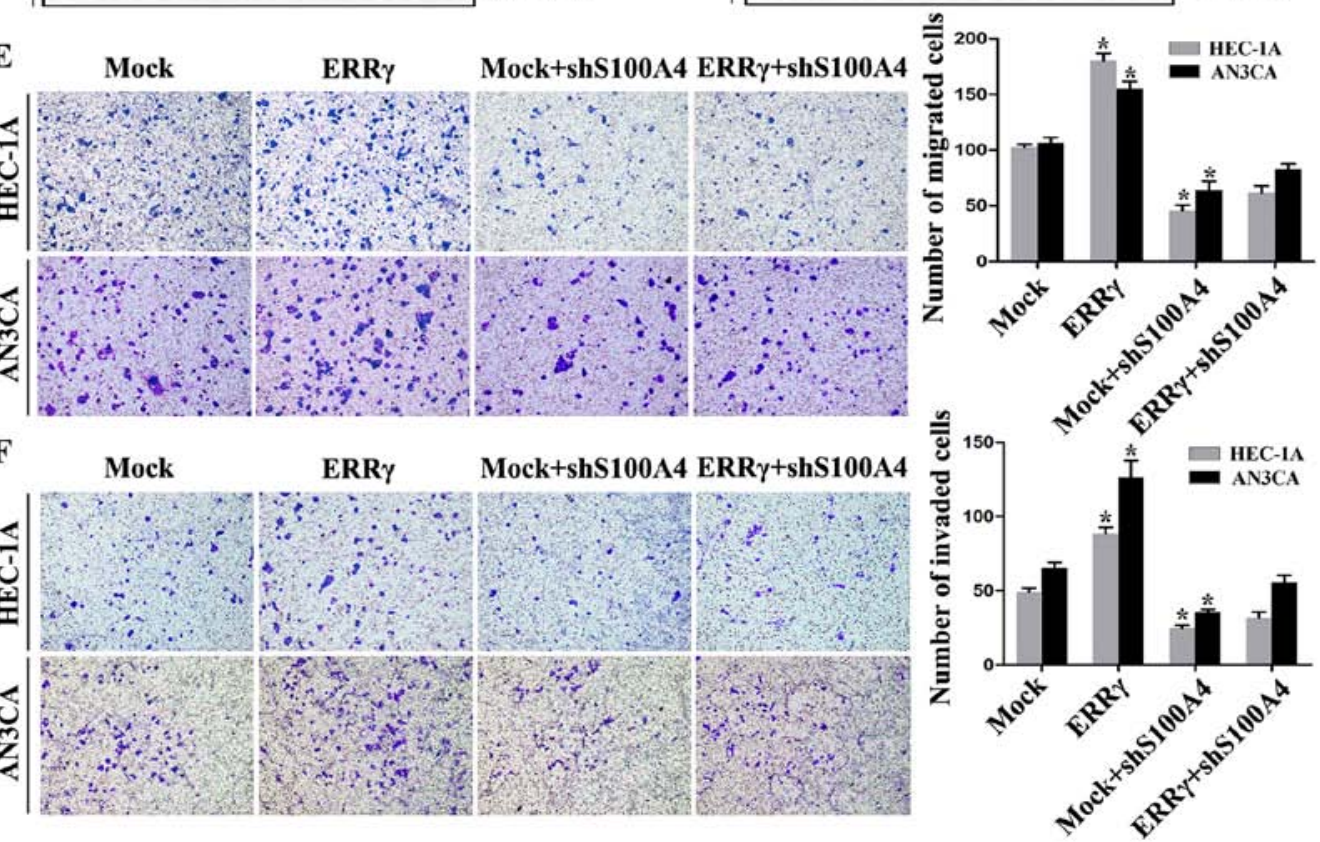

$\mathbf{F}$

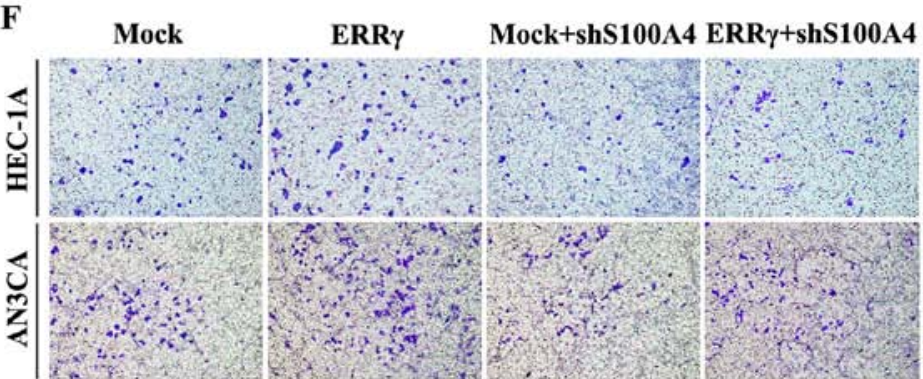

Figure 4. ERR $\gamma$ modulates the migration and invasion capability of EC cells through S100A4 in vitro. (A) Western blotting showing the protein levels of ERR $\gamma$ and S100A4 in EC cells stably transfected with sh-Scb, sh-ERR $\gamma$ or co-transfected with S100A4 expression vector. (B) Representation of migrated and invaded HEC-1B cells upon transfection with sh-Scb, sh-ERR $\gamma$ or co-transfected with S100A4 revealed with Transwell assays after $24 \mathrm{~h}$. (C and D) Western blotting showing the protein levels of ERR $\gamma$ and S100A4 in EC cells stably transfected with empty vector (mock), ERR $\gamma$ and co-transfected with sh-S100A4. (E and F) Representation (left) and quantification (right) of migrated and invaded HEC-1A and AN3CA cells upon transfection with mock, ERR $\gamma$, or co-transfection with sh-S100A4 determined with Transwell assays after $24 \mathrm{~h}$. "P<0.01. S100A4, S100 calcium binding protein A4; ERR $\gamma$, estrogen-related receptor $\gamma$. 
A

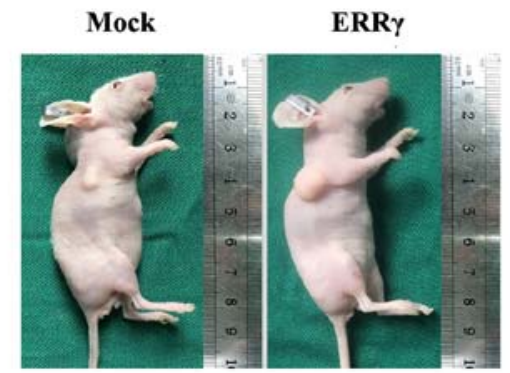

B

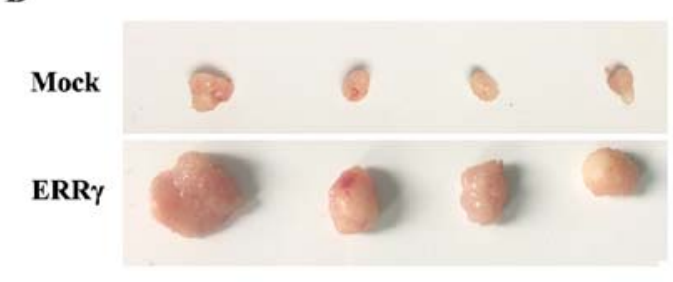

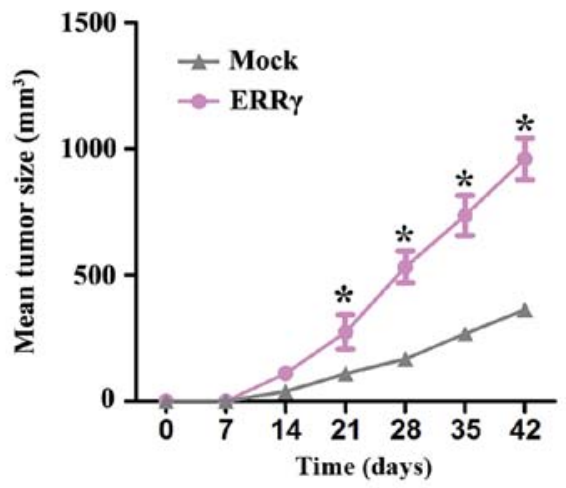

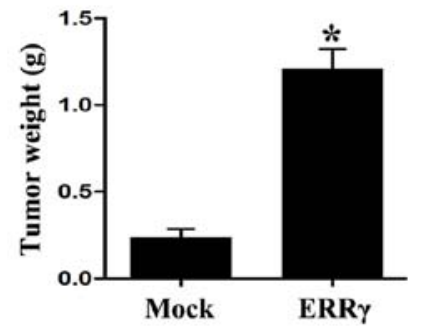

C

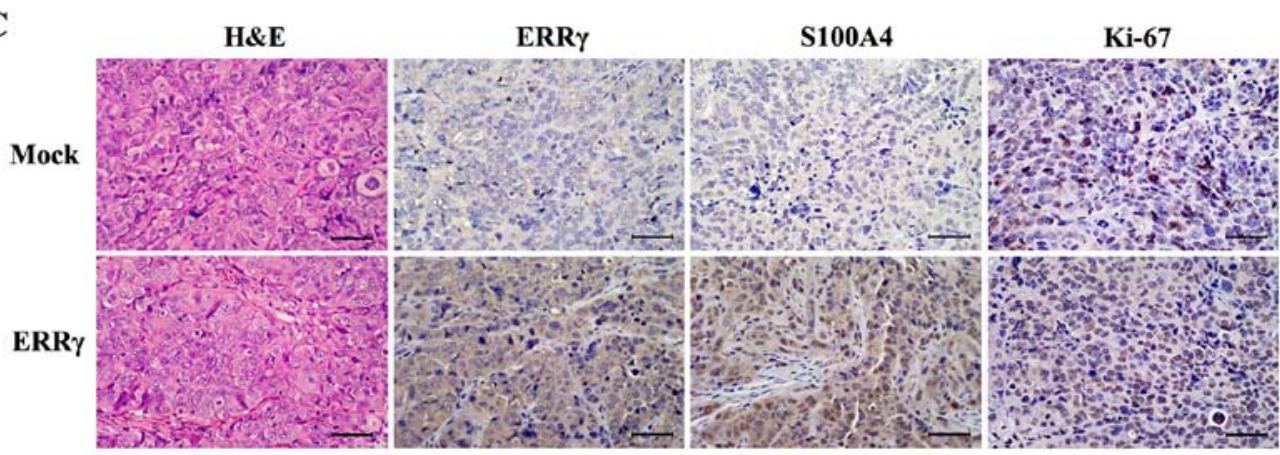

Figure 5. ERR $\gamma$ promotes the growth of EC cells in vivo. (A) Tumor growth curve of HEC-1A cells $\left(5 \times 10^{6}\right)$ stably transfected with ERR $\gamma$ or empty vector (mock) injected into athymic nude mice ( $\mathrm{n}=5$ for each group). (B) Representation (left) and quantification (right) of xenograft tumors formed by injection of HEC-1A cells stably transfected with mock or ERR $\gamma$. (C) The hematoxylin and eosin and immunohistochemical analyses of xenografts. ERR $\gamma$, S100A4 and Ki-67 expression were verified in the mock and intervention groups. Scale bars, $50 \mu \mathrm{m}$. "P<0.01. S100A4, S100 calcium binding protein A4; ERR $\gamma$, estrogen-related receptor $\gamma$.

ERR $\gamma$ knockdown and S100A4 restoration on the migration and invasion capacity of EC cells. ERR $\gamma$ knockdown decreased the expression of S100A4, and ectopic expression of S100A4 restored the ERR $\gamma$ knockdown-induced S100A4 downregulation in HEC-1B cells (Fig. 4A). In Transwell migration assays, ERR $\gamma$ knockdown inhibited the migration capability of HEC-1B cells compared to that of cells transfected with sh-Scb. Matrigel invasion assays showed that HEC-1B cells stably transfected with sh-ERR $\gamma$ presented an impaired invasion capacity compared to sh-Scb group cells. In addition, restoration of S100A4 expression rescued the EC cells from the defects in migration and invasion capabilities induced by ERR $\gamma$ downregulation (Fig. 4B). These results revealed that S100A4 was involved in ERR $\gamma$ knockdown-induced EC cell migration and invasion inhibition.

The impacts of ERR $\gamma$ overexpression and S100A4 restoration on cultured EC cells were further studied. Transfection of HEC-1A and AN3CA cells with sh-S100A4 resulted in reduced S100A4 protein levels and restored the upregulation of S100A4 induced by ERR $\gamma$ (Fig. 4C and D). In Transwell migration assays, ectopic ERR $\gamma$ expression increased the migration capability of HEC-1A and AN3CA cells compared with cells transfected with empty vector (mock) (Fig. 4E). Matrigel invasion assays revealed that EC cells stably transfected with ERR $\gamma$ exhibited an enhanced invasion capacity compared with mock group cells (Fig. 4F). Moreover, restoration of S100A4 expression prevented the enhanced migration and invasion capacity in EC cells induced by stable overexpression of ERR $\gamma$ (Fig. 4E and F). These findings suggest that S100A4 could, at least in part, mediate ERR $\gamma$-induced promotion of EC cell aggressiveness.

$E R R \gamma$ promotes the growth of EC cells in vivo. The efficacy of ERR $\gamma$ overexpression on tumor growth in vivo was further investigated. HEC-1A cells with fixed ERR $\gamma$ expression were subcutaneously injected into nude mice, leading to an increased proliferative index and tumor weight compared with tumors formed from cells transfected with the empty vector (Fig. 5A and B). Immunohistochemical analysis also showed that the expression of ERR $\gamma$ and its downstream gene 


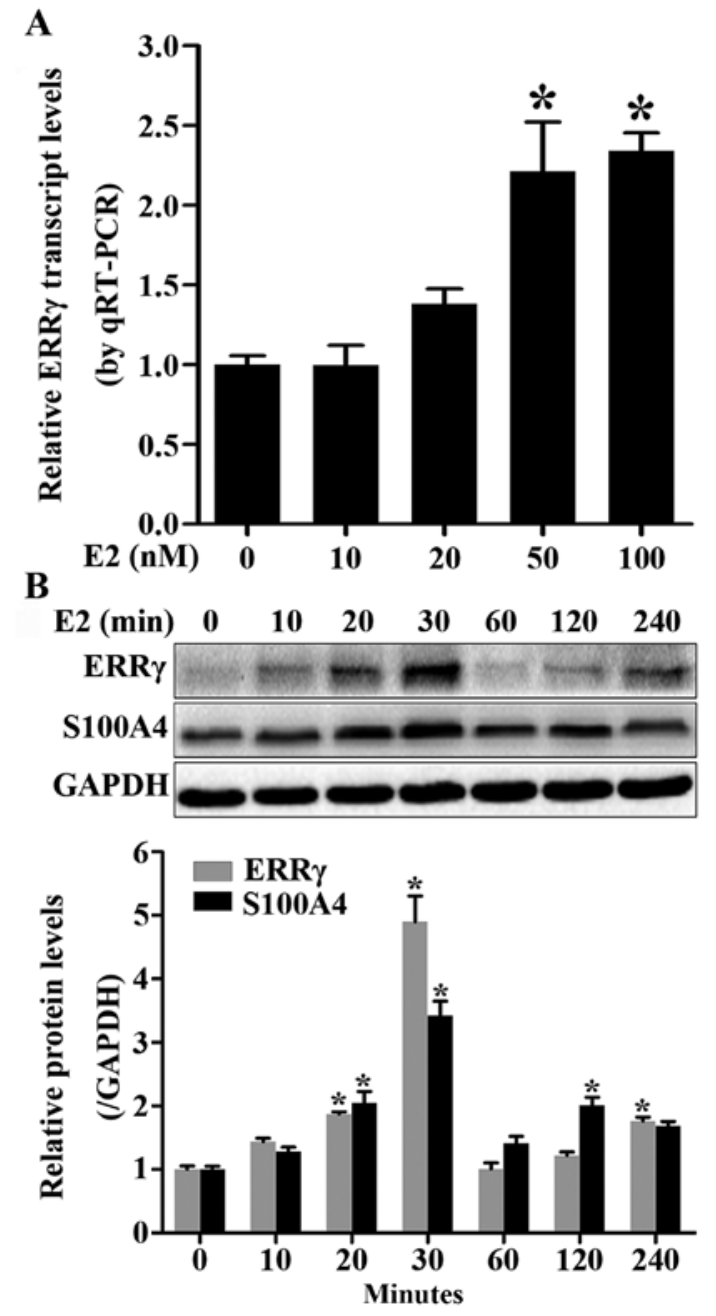

Figure 6. Upregulation of ERR $\gamma$ and S100A4 in estrogen-treated HEC-1A cells. (A) Transcription levels of ERR $\gamma$ in HEC-1A cells treated with 0,10 , 20,50 and $100 \mathrm{nM}$ of estrogen for $24 \mathrm{~h}$. (B) Time course of ERR $\gamma$ and S100A4 protein and mRNA levels in HEC-1A cells treated with $50 \mathrm{nM}$ of estrogen for 0, 10, 20, 30, 60, 120 and 240 min. ${ }^{*} \mathrm{P}<0.01$. S100A4, S100 calcium binding protein A4; ERR $\gamma$, estrogen-related receptor $\gamma$.

S100A4 were increased by stable transfection with ERR $\gamma$. Notably, the cell proliferation marker Ki-67 was also upregulated in HEC-1A cells (Fig. 5C). These results revealed an oncogenic role of ERR $\gamma$ in EC and were consistent with the in vitro studies.

ERR $\gamma$ and S100A4 are upregulated in estrogen-treated $H E C-1 A$ cells. Since estrogen is a major factor in EC pathogenesis and progression, we hypothesized that estrogen may affect the expression of ERR $\gamma$ and S100A4 in EC cells. We first manipulated HEC-1A cells with increasing estrogen concentrations $(0,10,20,50$ and $100 \mathrm{nM})$ for $24 \mathrm{~h}$. In real-time quantitative RT-PCR assays, ERR $\gamma$ mRNA was significantly increased when estrogen concentration reached $50 \mathrm{nM}$, and no differences were detected between the 50- and 100-nM groups (Fig. 6A). We next treated HEC-1A cells with $50 \mathrm{nM}$ of estrogen for increasing durations $(0,10,20,30,60,120$ and $240 \mathrm{~min}$ ). Western blot analysis revealed that there was a time-dependent diversification in the protein levels of both ERR $\gamma$ and S100A4, and both reached a peak at $30 \mathrm{~min}$ (Fig. 6B). Furthermore, S100A4 levels increased almost coincidently with ERR $\gamma$, and a positive correlation was detected (correlation coefficient $\mathrm{R}=0.886, \mathrm{P}=0.0079$ ). Mining the public $\mathrm{GEO}$ database (GSE11869) revealed that a positive correlation between ERR $\gamma$ and S100A4 expression has also been found elsewhere in several types of EC cells after estrogen stimulation (correlation coefficient $\mathrm{R}=0.448, \mathrm{P}<0.0001$ ). The above findings suggest that estrogen may be an upstream regulator of ERR $\gamma$ and S100A4 expression in EC.

\section{Discussion}

It is generally accepted that S100A4 has profound impacts on numerous types of cancers, including EC, and S100A4 upregulation results in tumor progression and aggressiveness. Additionally, overexpression of S100A4 is a predictive indicator of metastasis and poor survival of cancer patients (17-20). Our previous studies demonstrated that S100A4 promoted endometrial cancer (EC) cell aggressiveness via EMT-related modifications (9). However, the regulatory mechanisms essential for S100A4 expression in EC remain largely unknown. Studies have suggested that tyrosine-protein kinase erbB 2 (ERBB2) receptor signaling and integrin signaling regulate $\mathrm{S} 100 \mathrm{~A} 4$ expression in human medulloblastoma and breast cancer cells $(21,22)$. More importantly, S100A4 gene expression can be regulated at the transcriptional level, because its promoter contains several putative regulatory elements for transcription factors. In colorectal cancer (CRC), functionally active $\beta$-catenin is indispensable for induction of S100A4 expression and results in enhanced S100A4-induced migration and invasion (23). An electrophoretic mobility shift assay (EMSA) and chromatin immunoprecipitation (ChIP) assay further confirmed binding of $\beta$-catenin to the S100A4 promoter (7). In the present study, synthetic approaches were employed to analyze transcription profiling of several cancer specimens and transcription factor binding reported in public databases and identified ERR $\gamma$ as a crucial modulator facilitating S100A4 expression in EC. Notably, ERR $\gamma$ is highly expressed and positively correlated with S100A4 levels in several types of cancers, including EC specimens.

Early works regarding ERR $\gamma$ and malignancy mainly focused on the potential crosstalk of ERR $\gamma$ with the classical estrogen pathway and excavating its master regulation role in energy metabolism (24-26). ERR $\gamma$ may exert oncogenic or tumor suppressive functions with tumor specificity. High ERR $\gamma$ expression is correlated with more favorable clinical outcomes in ovarian, breast, and prostate cancer, indicating its tumor-suppressing function in these cancers $(13,27,28)$. Conversely, ERR $\gamma$-positive staining in hepatocellular carcinoma (HCC) specimens was remarkably higher than that in adjacent non-tumor liver tissues and was associated with advanced clinical stage and pathological grade, and knocking down ERR $\gamma$ inhibited HCC cell proliferation and induced G1-phase arrest (29). In human EC, ERR $\gamma$ is expressed in $\sim 31.3 \%$ of EC tissues, and its immunoreactivity was correlated with worse progression-free survival and overall survival. Interestingly, the opposite EC cell responsiveness was observed under forced ERR $\gamma$ expression or estrogen stimulation with ER $\alpha$ status dependence (15). In addition, the transcription levels of ERR $\gamma$ in EC were increased with clinical staging, myometrial invasion, and metastatic lymph nodes. Inhibition 
of ERR $\gamma$ activity attenuated estrogen-induced proliferation of EC cells through AKT and ERK1/2 signaling abolition (16). However, the exact biological functions of ERR $\gamma$ in EC have never been explored. By means of gain- and loss-of-function studies, we demonstrated that ERR $\gamma$ facilitated migration and invasion of EC cells in vitro and promoted tumor growth in vivo, suggesting an oncogenic role of ERR $\gamma$ during EC progression.

Strict binding site specificity experiments indicated that the 3 members of ERRs preferentially recognize almost identical DNA elements, distinct from the traditional estrogen receptor element (ERE), referred to as the estrogen-related receptor response element (ERRE; TnAAGGTCA) (10). Subsequent studies identified widespread distribution of ERR $\gamma$ targets, and transcriptionally active ERR $\gamma$ forms a heterodimer or homodimer that binds to the promoter of target genes, while ligand is unnecessary for ERR $\gamma$ activity $(30,31)$. Studies have confirmed that ERR $\gamma$ regulates target genes mainly involved in cellular metabolism, including tricarboxylic acid (TCA) cycle genes, fatty acid $\beta$-oxidation (FAO) genes, and electron transport chain (ETC) genes (32). Ectopic expression of ERR $\gamma$ enhanced oxidative phosphorylation in breast cancer cells, and the shift to oxidative metabolism attenuated breast cancer cell proliferation and tumor growth in vitro and in vivo (33). In addition, ERR $\gamma$ can also directly bind to genes involved in cell growth, such as p21 and p27. Consistent with the favorable role of ERR $\gamma$ in breast cancer, ERR $\gamma$ reprograms the genetic profiles of breast cancer cells in a manner characteristic of mesenchymal-to-epithelial transition, in which E-cadherin was activated by ERR $\gamma$ directly (34). The target genes of ERR $\gamma$ involved in initiation and aggressiveness of EC still warrant investigation. In the present study, we showed that ERR $\gamma$ facilitated transcription of S100A4 in EC cells via S100A4 promotor activation. Furthermore, since restoration of S100A4 expression rescued EC cells from ERR $\gamma$-induced phenotype changes in aggressiveness, ERR $\gamma$ may exert its oncogenic functions by activating S100A4 transcription in EC.

Estrogen is not a natural ligand for ERR $\gamma$, as indicated by ligand binding studies and transfection experiments with reporter genes. However, ERR $\gamma$ stimulates ERE-mediated transcription and functions as an estrogen responsive gene in breast cancer cells. Estrogen exposure resulted in ERR $\gamma$ overexpression or translocation from the cytoplasm to the nucleus in breast cancer and EC, and ERR $\gamma$ further mediates the cell proliferation promotion effects induced by estrogen $(16,35)$. Apart from the crucial role of estrogen in cell growth, emerging evidence has indicated the involvement of estrogen in cell aggressiveness in certain types of cancers, such as breast, ovarian and EC, partially through cell stemness, motility and EMT promotion $(36,37)$. In the present study, we found that ERR $\gamma$ expression is stimulated dose- and time-dependently by estrogen in HEC-1A EC cells. In addition, ERR $\gamma$ expression is unexpectedly correlated with S100A4 after different times of estrogen exposure. With consistent data from public datasets, we suspect that ERR $\gamma$ may mediate estrogen signaling in EC progression by modulating S100A4 expression, which warrants further investigation.

In conclusion, for the first time, this study demonstrates that ERR $\gamma$ is upregulated and positively related to S100A4 expression in EC. Additionally, ERR $\gamma$ facilitates S100A4 transcription through promoter activation and promotes the migration and invasion capability of EC cell lines. Furthermore, the expression of both ERR $\gamma$ and S100A4 could be regulated by estrogen stimulation. These findings extend our current knowledge of the mechanism of S100A4 regulation by transcription factors and suggest that ERR $\gamma$ could be a potential novel therapeutic target in human EC.

\section{Acknowledgements}

Not applicable.

\section{Funding}

The present study was supported by the National Natural Science Foundation of China (grant no. 81601260).

\section{Availability of data and materials}

The datasets used during the present study are available from the authors upon reasonable request.

\section{Authors' contributions}

HBW, YCZ and TH conceived and designed the study. TH, XXW, SQC and YL performed the experiments. DLF and YCZ were involved in data analysis. TH and XXW wrote the paper. HBW, YCZ and DLF reviewed and edited the manuscript. All authors read and approved the manuscript and agree to be accountable for all aspects of the research in ensuring that the accuracy or integrity of any part of the work are appropriately investigated and resolved.

\section{Ethics approval and consent to participate}

The present study was performed in accordance with the Declaration of Helsinki, and approval to conduct the present study was obtained from the Ethics Committee of Tongji Medical College, Huazhong University of Science and Technology (IORG no: IORG0003571). Tissues were collected after receiving informed consent from the patients. All animal experiments were approved by the Animal Care Committee of Tongji Medical College.

\section{Patient consent for publication}

Not applicable.

\section{Competing interests}

The authors state that they have no competing interests.

\section{References}

1. Morice P, Leary A, Creutzberg C, Abu-Rustum N and Darai E: Endometrial cancer. Lancet 387: 1094-1108, 2016.

2. Siegel RL, Miller KD and Jemal A: Cancer Statistics, 2017. CA Cancer J Clin 67: 7-30, 2017.

3. Makker A and Goel MM: Tumor progression, metastasis, and modulators of epithelial-mesenchymal transition in endometrioid endometrial carcinoma: An update. Endocr Relat Cancer 23: R85-R111, 2016. 
4. Donato R, Cannon BR, Sorci G, Riuzzi F, Hsu K, Weber DJ and Geczy CL: Functions of S100 proteins. Curr Mol Med 13: 24-57, 2013.

5. Stewart RL and O'Connor KL: Clinical significance of the integrin $\alpha 6 \beta 4$ in human malignancies. Lab Invest 95: 976-986, 2015

6. Kiss B, Kalmar L, Nyitray L and Pal G: Structural determinants governing S100A4-induced isoform-selective disassembly of nonmuscle myosin II filaments. FEBS J 283: 2164-2180, 2016.

7. Dahlmann M, Kobelt D, Walther W, Mudduluru G and Stein U: S100A4 in cancer metastasis: Wnt signaling-driven interventions for metastasis restriction. Cancers 8: pii: E59, 2016.

8. Bresnick AR, Weber DJ and Zimmer DB: S100 proteins in cancer. Nat Rev Cancer 15: 96-109, 2015.

9. Hua T, Liu S, Xin X, Cai L, Shi R, Chi S, Feng D and Wang H: S100A4 promotes endometrial cancer progress through epithelial-mesenchymal transition regulation. Oncol Rep 35: 3419-3426, 2016

10. Deblois G and Giguere V: Functional and physiological genomics of estrogen-related receptors (ERRs) in health and disease. Biochim Biophys Acta 1812: 1032-1040, 2011.

11. Ranhotra HS: The orphan estrogen-related receptor alpha and metabolic regulation: New frontiers. J Recept Signal Transduct Res 35: 565-568, 2015.

12. Madhavan S, Gusev Y, Singh S and Riggins RB: ERRgamma target genes are poor prognostic factors in Tamoxifen-treated breast cancer. J Exp Clin Cancer Res 34: 45, 2015.

13. Misawa A and Inoue S: Estrogen-Related Receptors in Breast Cancer and Prostate Cancer. Front Endocrinol 6: 83, 2015.

14. Gao M, Sun P, Wang J, Zhao D and Wei L: Expression of estrogen receptor-related receptor isoforms and clinical significance in endometrial adenocarcinoma. Int J Gynecol Cancer 16: 827-833, 2006.

15. Yamamoto T, Mori T, Sawada M, Kuroboshi H, Tatsumi $\mathrm{H}$, Yoshioka T, Matsushima H, Iwasaku K and Kitawaki J: Estrogen-related receptor-gamma regulates estrogen receptor-alpha responsiveness in uterine endometrial cancer. Int J Gynecol Cancer 22: 1509-1516, 2012.

16. Sun Y, Wang C, Yang H and Ma X: The effect of estrogen on the proliferation of endometrial cancer cells is mediated by ERRgamma through AKT and ERK1/2. Eur J Cancer Prev 23: 418-424, 2014.

17. Huang S, Zheng J, Huang Y, Song L, Yin Y, Ou D, He S, Chen X and Ouyang X: Impact of S100A4 expression on clinicopathological characteristics and prognosis in pancreatic cancer: A meta-analysis. Dis Markers 2016: 8137378, 2016.

18. Ling $\mathrm{Z}$ and $\mathrm{Li}$ R: Clinicopathological and prognostic value of S100A4 expression in gastric cancer: A meta-analysis. Int J Biol Markers 29: e99-e111, 2014.

19. Stewart RL, Carpenter BL, West DS, Knifley T, Liu L, Wang C, Weiss HL, Gal TS, Durbin EB, Arnold SM, et al: S100A4 drives non-small cell lung cancer invasion, associates with poor prognosis, and is effectively targeted by the FDA-approved anti-helminthic agent niclosamide. Oncotarget 7: 34630-34642, 2016.

20. Dou C, Liu Z, Xu M, Jia Y, Wang Y, Li Q, Yang W, Zheng X, Tu K and Liu Q: miR-187-3p inhibits the metastasis and epithelial-mesenchymal transition of hepatocellular carcinoma by targeting S100A4. Cancer Lett 381: 380-390, 2016.

21. Hernan R, Fasheh R, Calabrese C, Frank AJ, Maclean KH, Allard D, Barraclough R and Gilbertson RJ: ERBB2 up-regulates S100A4 and several other prometastatic genes in medulloblastoma. Cancer Res 63: 140-148, 2003.

22. Kim TH, Kim HI, Soung YH, Shaw LA and Chung J: Integrin (alpha6beta4) signals through $\mathrm{Src}$ to increase expression of S100A4, a metastasis-promoting factor: Implications for cancer cell invasion. Mol Cancer Res 7: 1605-1612, 2009.
23. Wang H, Shi J, Luo Y, Liao Q, Niu Y, Zhang F, Shao Z, Ding Y and Zhao L: LIM and SH3 protein 1 induces TGFbeta-mediated epithelial-mesenchymal transition in human colorectal cancer by regulating S100A4 expression. Clin Cancer Res 20: 5835-5847, 2014.

24. Huss JM, Garbacz WG and Xie W: Constitutive activities of estrogen-related receptors: Transcriptional regulation of metabolism by the ERR pathways in health and disease. Biochim Biophys Acta 1852: 1912-1927, 2015.

25. Deblois G, St-Pierre J and Giguere V: The PGC-1/ERR signaling axis in cancer. Oncogene 32: 3483-3490, 2013.

26. Giguere V: To ERR in the estrogen pathway. Trends Endocrinol Metab 13: 220-225, 2002

27. Sun P, Sehouli J, Denkert C, Mustea A, Könsgen D, Koch I, Wei L and Lichtenegger W: Expression of estrogen receptor-related receptors, a subfamily of orphan nuclear receptors, as new tumor biomarkers in ovarian cancer cells. J Mol Med 83: 457-467, 2005.

28. Audet-Walsh E, Yee T, McGuirk S, Vernier M, Ouellet C, St-Pierre $J$ and Giguère V: Androgen-dependent repression of errgamma reprograms metabolism in prostate cancer. Cancer Res 77: 378-389, 2017.

29. Kim JH, Choi YK, Byun JK, Kim MK, Kang YN, Kim SH, Lee S, Jang BK and Park KG: Estrogen-related receptor gamma is upregulated in liver cancer and its inhibition suppresses liver cancer cell proliferation via induction of p21 and p27. Exp Mol Med 48: e213, 2016.

30. Huppunen J and Aarnisalo P: Dimerization modulates the activity of the orphan nuclear receptor ERRgamma. Biochem Biophys Res Commun 314: 964-970, 2004.

31. Greschik H, Wurtz JM, Sanglier S, Bourguet W, van Dorsselaer A, Moras D and Renaud JP: Structural and functional evidence for ligand-independent transcriptional activation by the estrogen-related receptor 3. Mol Cell 9: 303-313, 2002.

32. Audet-Walsh E and Giguere V: The multiple universes of estrogen-related receptor $\alpha$ and $\gamma$ in metabolic control and related diseases. Acta Pharmacol Sin 36: 51-61, 2015.

33. Eichner LJ, Perry MC, Dufour CR, Bertos N, Park M, St-Pierre J and Giguère V: miR-378* mediates metabolic shift in breast

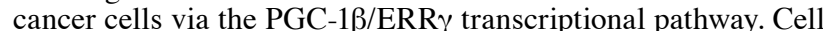
Metab 12: 352-361, 2010.

34. Tiraby C, Hazen BC, Gantner ML and Kralli A: Estrogen-related receptor gamma promotes mesenchymal-to-epithelial transition and suppresses breast tumor growth. Cancer Res 71: 2518-2528, 2011.

35. Ijichi N, Shigekawa T, Ikeda K, Horie-Inoue K, Fujimura T, Tsuda H, Osaki A, Saeki T and Inoue S: Estrogen-related receptor $\gamma$ modulates cell proliferation and estrogen signaling in breast cancer. J Steroid Biochem Mol Biol 123: 1-7, 2011.

36. Jeon SY, Hwang KA and Choi KC: Effect of steroid hormones, estrogen and progesterone, on epithelial mesenchymal transition in ovarian cancer development. J Steroid Biochem Mol Biol 158: $1-8,2016$

37. Sun Y, Wang Y, Fan C, Gao P, Wang X, Wei G and Wei J: Estrogen promotes stemness and invasiveness of ER-positive breast cancer cells through Gli1 activation. Mol Cancer 13: 137, 2014.

38. Day RS, McDade KK, Chandran UR, Lisovich A, Conrads TP, Hood BL, Kolli VS, Kirchner D, Litzi T and Maxwell GL: Identifier mapping performance for integrating transcriptomics and proteomics experimental results. BMC Bioinformatics 12: 213, 2011

39. Pappa KI, Polyzos A, Jacob-Hirsch J, Amariglio N, Vlachos GD, Loutradis D and Anagnou NP: Profiling of discrete gynecological cancers reveals novel transcriptional modules and common features shared by other cancer types and embryonic stem cells. PLoS One 10: e0142229, 2015. 